\title{
Exploitation des données dosimétriques, les dossiers médicaux, SISERI
}

\author{
A. BIAU
}

Dès les années 1990, à la création de l'OPRI, une de ses premières missions a été de constituer une base de données dosimétriques nationales permettant de suivre plus facilement les doses des travailleurs durant leur carrière. Le besoin a été accru par la demande d'études épidémiologiques et l'avènement de la dosimétrie opérationnelle obligatoire dont les résultats permettaient un suivi au jour le jour.

\subsection{Transmission des données et bilans annuels}

Le SCPRI, puis l'OPRI et enfin l'IRSN ont, dès l'origine de la réglementation en matière de suivi dosimétrique des travailleurs, eu pour mission de centraliser les résultats de la dosimétrie individuelle pour contrôler la conformité des doses individuelles aux limites réglementaires, mais au fil du temps, cette mission s'est élargie et adaptée aux évolutions de la technique et du ressenti sociologique relatif aux risques liés à l'exposition aux rayonnements ionisants.

Les résultats du SCPRI et ceux transmis par les autres laboratoires (LCIE essentiellement) au SCPRI chaque mois étaient compilés sous forme informatique en fonction de l'état de l'art informatique, cartes perforées, puis disquettes et CD.

En fin d'année, les doses étaient éditées sur des listings appelés «cumuls annuels » portant pour chaque travailleur les 12 doses et la dose cumulée sur l'année civile. À noter que jusqu'au décret de 1986, la limite de dose portait sur l'année civile et non pas sur 12 mois glissants.

Ces listings étaient reliés pour former un document auquel on pouvait se référer pour faire des bilans globaux ou reconstituer des « passés dosimétriques » lorsque les résultats originaux étaient manquants dans le dossier médical tenu par le médecin du travail.

Ces reconstitutions dosimétriques faites en général à la demande de médecins du travail étaient fréquentes et jusque dans des années récentes, à titre d'exemple, plus de 400 reconstitutions ont été réalisées en 1999. 
À l'origine, les bilans globaux portaient sur les doses annuelles pour l'ensemble des travailleurs mais il n'y avait pas de distribution par type d'activité professionnelle. En 1973, Jean-Pierre Moroni, chef du Département de radiophysique auquel appartenait le laboratoire de dosimétrie individuelle m'a confié la tâche de codifier chaque établissement abonné au SCPRI en fonction de sa nature et du type d'appareil émetteur de rayonnement utilisé.

Les 5000 établissements abonnés à cette époque ont été classés en 47 catégories, à partir de l'analyse de leur dossier d'inscription, selon un code comprenant un caractère alphanumérique pour le type d'établissement et un autre pour le type d'appareil. Par la suite, chaque nouvel établissement abonné se voyait attribuer un code «statistique » selon la même codification.

Ainsi à partir de 1975, le SCPRI a publié, chaque année, un bilan statistique des doses annuelles de ses abonnés.

Ces bilans étaient incomplets car ils ne concernaient que les abonnés du SCPRI mais aussi parce que la codification elle-même, faite au niveau des établissements et non par individu, n'était pas parfaite. On considérait en effet, pour simplifier, qu'au sein d'un établissement tous les abonnés faisaient le même travail, ce qui n'était pas toujours le cas lorsque plusieurs types de sources de rayonnements coexistaient. Malgré les imperfections signalées, ces premiers bilans ont été transmis chaque année au comité scientifique des Nations unies sur l'étude des effets et des niveaux des rayonnements ionisants (UNSCEAR: United Nations Scientific Committee on the Effects of Atomic Radiations).

Les laboratoires des exploitants nucléaires établissaient également leurs propres statistiques également transmises à l'UNSCEAR. Cet organisme publie tous les cinq ans un rapport comprenant une synthèse des résultats fournis par les différents pays sur l'exposition des travailleurs et des patients mais également sur les niveaux de radioactivité naturelle et artificielle dans l'environnement et donc de l'exposition du public.

Au fil des années, la transmission et l'exploitation des données s'est améliorée. Pour sa part, le LCIE a adopté une codification plus simple que celle du SCPRI mais qui permettait d'agréger les données des deux laboratoires. Par la suite, on a pu intégrer les données de Philips (ex Massiot).

À titre d'exemple les tableaux I et II montrent la distribution détaillée des doses reçues dans les différents types d'activité en dehors des installations nucléaires de base (INB) pour les abonnés suivis par l'OPRI, le LCIE et Philips (tableau I). En 2000, 176967 personnes ont été suivies par ces trois laboratoires en milieu non nucléaire dont 115028 par l'OPRI (65\%), 30973 par le LCIE (22\%) et 23006 par Philips (22\%). 
TABLEAU I

Répartition des doses en milieu non nucléaire en 2000 (données OPRI, LCIE et Philips) (OPRI, 2001).

\begin{tabular}{lccccccc}
\hline Secteur d'activité & $\begin{array}{c}\text { Nombre de } \\
\text { personnes }\end{array}$ & $\begin{array}{c}\text { Doses } \\
<1 \mathrm{mSv}\end{array}$ & $\begin{array}{c}\text { Doses } \\
1-6 \mathrm{mSv}\end{array}$ & $\begin{array}{c}\text { Doses } \\
6-15 \mathrm{mSv}\end{array}$ & $\begin{array}{c}\text { Doses } \\
15-20 \mathrm{mSv} \text { v }\end{array}$ & $\begin{array}{c}\text { Doses } \\
>20 \mathrm{mSv}\end{array}$ & $\begin{array}{c}\text { Dose collective } \\
\text { Homme.Sv }\end{array}$ \\
\hline Radiologie & 90621 & 89129 & 1156 & 263 & 56 & 17 & 11,50 \\
Radiothérapie & 7944 & 7701 & 193 & 42 & 5 & 3 & 1,53 \\
$\begin{array}{l}\text { Médecine } \\
\text { Nucléaire }\end{array}$ & 4046 & 3606 & 396 & 44 & 0 & 0 & 1,56 \\
Sources in vitro & 3357 & 3305 & 52 & 0 & 0 & 0 & 0,13 \\
Dentaire & 24752 & 24570 & 140 & 33 & 7 & 2 & 1,20 \\
Médecine sociale & 5312 & 5288 & 19 & 2 & 1 & 2 & 0,33 \\
Vétérinaires & 3782 & 3755 & 18 & 7 & 2 & 0 & 0,22 \\
Radiographie & 23725 & 19067 & 3145 & 1461 & 50 & 2 & 21,24 \\
industrielle X et $\gamma$ & & & & & & & 0,15 \\
Recherche & 7794 & 7759 & 31 & 4 & 0 & 0 & 0,64 \\
Divers & 5634 & 5551 & 58 & 24 & 0 & 1 & 38,51 \\
Total & 176967 & 169731 & 5208 & 1880 & 121 & 27 & \\
\hline
\end{tabular}

On observe que la radiologie médicale, au sens large, qui comprend la radiographie classique et interventionnelle, ainsi que les scanners, présente le plus gros effectif et le plus grand nombre de doses supérieures à $20 \mathrm{mSv}$. L'autre poste important est la radiographie industrielle $X$ et $\gamma$ qui représente un effectif quatre fois moindre, huit fois moins de doses supérieures à $20 \mathrm{mSv}$ mais une dose totale presque double $(21,24$ Homme.Sv contre 11,5$)$ qui montre que le pourcentage de personnels exposés est plus important; l'expérience montre que c'est essentiellement dans le domaine de la radiographie $\gamma$ avec des sources scellées d'iridium 192 ou de cobalt 60 que les doses sont les plus élevées.

Le tableau II présente la répartition des doses des personnels travaillant dans les installations nucléaires. Les résultats de dosimétrie individuelle proviennent de l'OPRI et du LCIE pour les agents d'entreprises extérieures et pour les agents statutaires d'EDF, COGEMA, CEA, CNRS (IN2P3) et CERN par les laboratoires de dosimétrie de ces organismes.

Dans ce domaine également la situation est très diversifiée selon le type de personnel surveillé. Ainsi les agents d'entreprises extérieures représentent un effectif de $46 \%$ de l'effectif total, alors que le pourcentage en termes de dose totale est de $66 \%$. Pour les agents EDF le pourcentage en effectif est de $28 \%$ et en dose totale ce pourcentage est le même, ce qui montre que la dose moyenne est supérieure pour les agents d'entreprise, de même pour les dépassements de la dose de $20 \mathrm{mSv}$, 14 contre 1 . 
TABLEAU II

Répartition des doses en 2000 dans le milieu nucléaire (OPRI, 2001).

\begin{tabular}{|c|c|c|c|c|c|c|c|}
\hline Secteur d'activité & $\begin{array}{l}\text { Personne } \\
\text { s suivies }\end{array}$ & $\begin{array}{c}\text { Doses } \\
<1 \mathrm{mSv}\end{array}$ & $\begin{array}{c}\text { Doses } \\
1-6 \mathrm{mSv}\end{array}$ & $\begin{array}{c}\text { Doses } \\
6-15 \mathrm{mSv}\end{array}$ & $\begin{array}{c}\text { Doses } \\
15-20 \mathrm{mSV}\end{array}$ & $\begin{array}{c}\text { Doses } \\
>20 \mathrm{mSv}\end{array}$ & $\begin{array}{c}\text { Dose collective } \\
\text { Homme.Sv }\end{array}$ \\
\hline $\begin{array}{l}\text { Centrales } \\
\text { nucléaires } \\
\text { Agents EDF }\end{array}$ & 23172 & 19180 & 3596 & 387 & 8 & 1 & 13,91 \\
\hline $\begin{array}{l}\text { Cycle du } \\
\text { combustible } \\
\text { Agents COGEMA } \\
\text { La Hague } \\
\text { Marcoule }\end{array}$ & $\begin{array}{l}2878 \\
3927\end{array}$ & $\begin{array}{l}2849 \\
3698\end{array}$ & $\begin{array}{c}29 \\
212\end{array}$ & $\begin{array}{c}0 \\
17\end{array}$ & $\begin{array}{l}0 \\
0\end{array}$ & $\begin{array}{l}0 \\
0\end{array}$ & $\begin{array}{l}0,15 \\
0,77\end{array}$ \\
\hline $\begin{array}{l}\text { Recherche } \\
\text { CEA } \\
\text { IN2P3 } \\
\text { CERN }\end{array}$ & $\begin{array}{l}7791 \\
3701 \\
2712\end{array}$ & $\begin{array}{l}7608 \\
3687 \\
2559\end{array}$ & $\begin{array}{c}178 \\
13 \\
140\end{array}$ & $\begin{array}{c}4 \\
1 \\
13\end{array}$ & $\begin{array}{l}1 \\
0 \\
0\end{array}$ & $\begin{array}{l}0 \\
0 \\
0\end{array}$ & $\begin{array}{l}0,91 \\
0,05 \\
0,62\end{array}$ \\
\hline $\begin{array}{l}\text { Entreprises } \\
\text { extérieures }\end{array}$ & 37769 & 30898 & 4793 & 1903 & 164 & 14 & 32,56 \\
\hline Total & 81950 & 70479 & 8958 & 2325 & 173 & 15 & 48,97 \\
\hline
\end{tabular}

Dans le domaine de la recherche, le pourcentage en effectif est de $17 \%$ et en dose totale ou collective de $3 \%$, ce qui montre que dans ce secteur l'exposition moyenne est très faible et il n'y a aucun dépassement de dose de $20 \mathrm{mSv}$.

Ces bilans, transmis chaque année au ministère du Travail, donnaient un aperçu complet de l'exposition des travailleurs, ils seront établis sur ces modèle jusqu'en 2009 ou 2010 lorsque le système SISERI (voir plus loin) permettra de les réaliser à partir de la base de données nominatives et non plus par intégration des bilans de chaque laboratoire.

\subsection{Le Système informatique de surveillance de l'exposition aux rayonnements ionisants (SISERI)}

En agrégeant les statistiques des laboratoires, il n'est pas exclu qu'un agent puisse être suivi dans la même année par deux laboratoires différents pour le compte de deux employeurs et dans ce cas, cet agent est compté deux fois avec deux doses non cumulées. Si l'on se limite à évaluer l'impact radiologique des différentes activités professionnelles présentant un risque d'exposition aux rayonnements ionisants, les bilans évoqués ci-dessus sont suffisants. Mais depuis les années 1980, avec la demande croissante d'études épidémiologiques, l'archivage simple des années 1970 et les bilans sans cesse améliorés entre les années 1980 et 1990 ne suffisent plus. De plus, l'avènement de la dosimétrie opérationnelle selon la Directive 90/64 EURATOM, a donné naissance au système DOSINAT, base de 
données recueillant quotidiennement les doses opérationnelles relevées dans les CNPE. Ce système DOSINAT d'EDF devait être élargi à un système DOSIMO pour le CEA et COGEMA. Ces systèmes ne concernaient que la dosimétrie opérationnelle mais ils ont rapidement montré leur efficacité en termes d'optimisation concrétisée par un abaissement significatif des doses reçues par les travailleurs de ces sites.

Parallèlement, pour avoir participé en tant que fournisseur de données dosimétriques à deux études épidémiologiques (Cardis et al., 2007 ; Guérin et al., 2009), il m'est apparu que les données étaient très incomplètes que ce soit dans les bases de données ou dans les dossiers médicaux. Ainsi en 1994, à la création de l'OPRI, des plans d'actions ont été lancés au ministère de la Santé (Bureau de la radioprotection) et j'ai été chargé d'animer un groupe de travail sur les bases de données dosimétriques.

Dans le contexte de la transcription des Directives 90/64 et 96/29 est apparue incontournable la création d'une base de données unique comportant, pour chaque agent clairement identifié, l'ensemble des données dosimétriques. C'est ainsi que le décret du 31 mars 2003, à l' article R 231-113, officialise la création d'un système permettant la centralisation, la consolidation et la conservation de l'ensemble des résultats des mesures individuelles de l'exposition des travailleurs.

La mise en place de SISERI a été une entreprise de longue haleine car il faut bien être conscient des bouleversements intervenus au cours de 40 dernières années sur les plans techniques et psychosociologiques. Du point de vue informatique, les progrès ont été considérables et selon les générations, on en est plus ou moins conscient. Dans les années 1960-1970, les moyens informatiques étaient primaires, cartes perforées, ruban perforés, mémoires limitées.

À tel point qu'en 1976, devant l'amoncellement des cartes perforées correspondant aux résultats dosimétriques individuels du SCPRI et du LCIE, il fut décidé de ne conserver que les résultats positifs (égaux ou supérieurs au seuil de mesure)... Cela ne changeait rien pour les bilans globaux mais lorsqu'un nom ne figurait pas dans le recueil de cumuls annuels on ne pouvait pas savoir si la personne n'avait eu que des doses nulles ou si elle n'avait pas porté son dosimètre et il fallait se retourner vers le médecin du travail destinataire de l'ensemble des résultats.

À partir de 1990, nous avons à nouveau conservé toutes les doses nulles ou positives et les supports de transfert des données par les laboratoires ont évolué vers des techniques plus modernes, disquette puis $\mathrm{CD}$.

Pour ce qui concerne le personnel du SCPRI, il faut se rappeler qu'à partir de 1985 il a disposé d'un Minitel (les cadres essentiellement) et qu'il a fallu attendre 2000 au temps de l'OPRI pour disposer d'ordinateurs de bureau fonctionnant en réseau. Cette situation était la même dans la plupart des organismes. Par contre, dès 
l'origine le SCPRI a disposé des moyens technologiques les plus modernes pour les mesures dosimétriques, spectrométriques et l'informatique scientifique.

Du point de vue du contexte sociétal, les notions d'épidémiologie n'étaient pas dans l'air du temps alors qu' aujourd'hui la société en demande à tout bout de champ dès qu'un phénomène n'est pas simplement expliqué. De même, la nécessité d'une base de données n'était pas évidente et il faut bien reconnaitre que le français moyen est a priori hostile à la mise en place de tout fichier nominatif.

Une base de données individuelles nécessite d'abord une excellente identification des personnes constituant cette base. Jusqu'en 1990 environ, l'identification des abonnés était sommaire. Au départ les formulaires d'abonnement ne demandaient que le nom, le prénom et les deux derniers chiffres de l'année de naissance, mais il n'y avait pas une rigueur excessive dans la saisie des informations. L'orthographe des noms n'était pas toujours exacte, le prénom était souvent limité à la première lettre, quant à l'année de naissance, elle était souvent manquante parce que, par exemple, la surveillante d'un grand service hospitalier qui remplissait le formulaire n'osait pas demander son année de naissance au chef de service ! Sachant que le médecin du travail devait tenir un dossier médical spécial, on considérait que ces imprécisions n'étaient pas dramatiques, l'essentiel étant que les dosimètres soient disponibles le plus vite possible. Plus tard, une plus grande rigueur a été requise et l'identification était complétée par les dix premiers chiffres du numéro de sécurité sociale et les nouveaux abonnés étaient eux-mêmes plus rigoureux dans leur demande. Toutefois, pour que l'identification soit parfaite il nous a paru indispensable de pouvoir disposer du numéro de sécurité sociale complet. C'est pourquoi, à partir de 1999 avec Jean-Luc Pasquier (Directeur scientifique de l'OPRI) nous avons travaillé avec la Commission nationale informatique et liberté (CNIL) pour obtenir l'autorisation d'utiliser ce numéro.

C'est ainsi qu'après avis du Conseil d'État et délibération de la CNIL en date du 24 juin 2004, est paru le décret n ${ }^{\circ}$ 2004-1489 du 30 décembre 2004 autorisant l'utilisation par l'IRSN du répertoire national d'identification des personnes physiques dans un traitement automatisé des données à caractère personnel relatives à la surveillance des travailleurs exposés aux rayonnements ionisants (voir chapitre 4).

Parallèlement, après une étude des besoins relatifs à la transmission et à la restitution des données, le projet de constitution du système SISERI a été lancé en 2000 avec un appel d'offres pour l'application informatique sous la responsabilité de Danièle Crescini et Gauzelin Barbier. 
Le système SISERI est devenu opérationnel en 2005 avec des conditions particulières d'accès nécessitant un certificat numérique et des règles précisées dans le tableau III. Depuis, les données ont sans cesse étaient complétées et améliorées avec notamment la création d'une nouvelle codification des activités qui permettra d'établir des statistiques plus précises sur l'exposition des travailleurs. Concrètement, SISERI est géré est animé par une équipe dirigée par Pascale Scanff au sein de la Direction de la radioprotection de l'homme (DRPH) de l'IRSN.

\section{TABLEAU III}

Règles d'accessibilité au système SISERI.

\begin{tabular}{ccccc}
\hline & \multicolumn{3}{c}{ Dose individuelle } \\
\hline & Opérationnelle & Passive & Interne & Efficace \\
\hline $\begin{array}{c}\text { PCR (accès } \\
\text { limité aux } \\
12 \text { derniers mois) }\end{array}$ & oui & non & non \\
$\begin{array}{c}\text { Médecins } \\
\text { du travail }\end{array}$ & oui & oui & oui & oui \\
\hline
\end{tabular}

+ Travailleur concerné : accès sur demande à tous ses résultats de dosimétrie.

+ Inspecteur du travail : accès sur demande aux doses opérationnelles et doses efficaces.

Actuellement, le système est performant surtout en ce qui concerne la dosimétrie opérationnelle. Pour la dosimétrie passive il reste à poursuivre la récupération complète des données anciennes.

\section{RÉFÉRENCES}

Cardis E., Vrijheid M., Blettner M. et al. (2007) The 15 Country Collaborative Study of Cancer Risk among Radiation workers in the Nuclear Industry: Estimates of Radiations Related Cancer risks, Radiation Research 167, 396-416.

Guérin S., Richard G., Biau A. et al. (2009) Cancer mortality among French nuclear contract workers, American Journal of Industrial Medicine $\mathbf{5 2}$. 